\title{
Mitigating the psychological effects of COVID-19 on health care workers
}

\author{
Peter E. Wu MD MSc, Rima Styra MD MEd, Wayne L. Gold MD
}

- Cite as: CMAJ 2020 april 27;192:E459-60. doi: 10.1503/cmaj.200519; early-released April 15, 2020

A Ithough tremendous efforts are being made to investigate the pathophysiology, clinical outcomes and treatment of coronavirus disease 2019 (COVID-19), the psychological effects of this pandemic on health care workers cannot be overlooked.

Experience from the 2003 severe acute respiratory syndrome (SARS) outbreak and early reports related to COVID-19 show that health care workers experience considerable anxiety, stress and fear. ${ }^{1,2}$ The psychological effects related to the current pandemic are driven by many factors, including uncertainty about the duration of the crisis, lack of proven therapies or a vaccine, and potential shortages of health care resources, including personal protective equipment. Health care workers are also distressed by the effects of social distancing balanced against the desire to be present for their families, and the possibility of personal and family illness. All of these concerns are amplified by the rapid availability of information and misinformation on the Internet and social media.

Health care workers may experience psychological distress from providing direct care to patients with COVID-19, knowing someone who has contracted or died of the disease, or being required to undergo quarantine or isolation. ${ }^{1-3}$ Mitigation strategies for all scenarios are vital to ensure psychological wellness and in turn ensure a healthy and robust clinical workforce.

Not surprisingly, providers caring for patients with COVID-19 are among those at greatest risk of psychologic distress. A survey of 1257 nurses and physicians caring for patients with the disease in China found that these providers (41.5\% of respondents) had significantly more depression, anxiety, insomnia and distress than providers who did not care directly for patients. ${ }^{1}$ Another observational study of 180 health care workers providing direct care for patients with COVID-19 found substantial levels of anxiety and stress that adversely influenced sleep quality and self-efficacy. ${ }^{4}$ Importantly, those who reported a strong social support network had a lower degree of stress and anxiety, and a higher level of self-efficacy. ${ }^{4}$ A qualitative study of medical residents during the 2003 SARS outbreak in Toronto showed that anxieties around personal safety and risk of contagion to loved ones conflicted with their professional duty to care. ${ }^{5}$ This highlights the complexity of issues faced by health care workers and

\section{KEY POINTS}

- Health care workers may experience considerable psychologic distress as a result of the COVID-19 pandemic due to providing direct patient care, vicarious trauma, quarantine or selfisolation.

- Strong leadership with clear, honest and open communication is needed to offset fears and uncertainties.

- Provision of adequate resources (e.g., medical supplies) and mental health supports will bolster individual self-efficacy and confidence.

- Leveraging online technology will allow delivery of psychosocial supports while preserving physical distancing.

- Emphasizing the altruism of working in health care and serving of the greater good will help health care workers to be reminded of their purpose in a time of crisis.

the dissonance they are required to reconcile. Providers not directly caring for patients with COVID-19 are not immune to psychologic effects and may have vicarious trauma at levels similar to the general public. ${ }^{6}$ It has been postulated that this may relate to their concerns for patients with the disease, their at-risk colleagues, ${ }^{6}$ and for themselves and their families.

A letter to the editor of Psychiatry and Clinical Neurosciences ${ }^{7}$ and a qualitative study conducted in a teaching hospital during the SARS outbreak ${ }^{8}$ described measures to mitigate the psychosocial impact on health care workers and identified themes that commonly arose in other studies exploring this issue. Clear and rapid hospital communication was helpful to address the reactions of health care workers based on uncertainty or fear. ${ }^{8}$ Frequent communication, without being overly reassuring, was identified as helpful. ${ }^{5,7,8}$ Leadership at the hospital level, infection prevention and control services, and other supervising bodies need to be transparent and flexible, acknowledge uncertainty and provide clear evidence-based plans that will bolster workers' trust, confidence and self-efficacy. This includes direction about hospital processes and appropriate provision of supplies and equipment, particularly in the face of potential shortages. ${ }^{2,5,7,8}$

Psychiatric support was offered to health care workers during the SARS outbreak, at first informally and then through confidential 


\section{Box 1: Resources for support of health care workers}

- Canadian Medical Association: Provincial Physician Health Program. Available: www.cma.ca/provincial-physician-health-program

- Canadian Psychological Association: Psychology works for COVID-19. Psychologists giving back to front line service providers. Available: https://cpa.ca/corona-virus/psychservices/

- Canadian Medical Association: Maintaining your and your family's well-being during a pandemic. Available: www.cma.ca/ maintaining-your-and-your-familys-well-being-during-pandemic

telephone lines and drop-in centres. ${ }^{8}$ The current need for physical distancing necessitates adjustments to these supportive interventions by leveraging today's technology (e.g., online video and audio capabilities). At the end of March 2020, mental health workers in Ontario established free confidential services via online registration for health care workers who care for patients with COVID-19. ${ }^{9}$ System-level changes (i.e., safe hospital policies and adequate resource provision) are likely to have more far-reaching effects than individual support, especially since capacity to counsel large numbers of affected health care workers may be limited. Box 1 lists select resources for health care workers who are seeking psychological support.

A rapid review of existing evidence found that health care workers who are self-isolating or under quarantine report symptoms of posttraumatic stress disorder, depression, stigmatization and fear of financial loss. ${ }^{3}$ Failure to ensure appropriate support could result in underreporting of symptoms and increase the risk of inhospital transmission from those who work while sick against advice. A strong social support network can offset feelings of isolation. ${ }^{3}$ Video calls and virtual meetings allow for maintenance of social relations while preserving physical distancing. Other mitigating interventions include delivery of general and medical supplies, limiting isolation to the shortest duration necessary, and emphasizing that altruism and serving of the greater good are core values of the profession. ${ }^{3}$ Medical students across Canada are showing altruism as they offer their services (e.g., running errands, delivering groceries and providing child care) to health care workers in need, ${ }^{10}$ as well as volunteering to assist with contact tracing. ${ }^{11}$ All these interventions can reduce the effect of quarantine or isolation and help to preserve wellness and fitness in health care workers so that they can return to work when able.

Supporting health care workers in all aspects is vital to sustaining a healthy workforce during the pandemic in Canada. Taking care of ourselves is vital so that we may continue to take care of others.

\section{References}

1. Lai J, Ma S, Wang Y, et al. Factors associated with mental health outcomes among health care workers exposed to coronavirus disease 2019. JAMA Netw Open 2020;3:e203976.

2. Styra R, Hawryluck L, Robinson S, et al. Impact on health care workers employed in high-risk areas during the Toronto SARS outbreak. J Psychosom Res 2008;64:177-83.

3. Brooks SK, Webster RK, Smith LE, et al. The psychological impact of quarantine and how to reduce it: rapid review of the evidence. Lancet 2020;395:912-20.

4. Xiao H, Zhang $\mathrm{Y}$, Kong D, et al. The effects of social support on sleep quality of medical staff treating patients with coronavirus disease 2019 (COVID-19) in January and February 2020 in China. Med Sci Monit 2020;26:e923549.

5. Rambaldini G, Wilson K, Rath $\mathrm{D}$, et al. The impact of severe acute respiratory syndrome on medical house staff: a qualitative study. J Gen Intern Med 2005;20: 381-5.

6. Li Z, Ge J, Yang M, et al. Vicarious traumatization in the general public, members, and non-members of medical teams aiding in COVID-19 control. Brain Behav Immun 2020 Mar. 10;pii:S0889-1591(20)30309-3.

7. Imai H. Trust is a key factor in the willingness of health professionals to work during the COVID-19 outbreak: experience from the H1N1 pandemic in Japan 2009 [letter]. Psychiatry Clin Neurosci 2020 Feb. 27. [Epub ahead of print]. doi: 10.1111/pcn.12995.

8. Maunder R, Hunter J, Vincent L, et al. The immediate psychological and occupational impact of the 2003 SARS outbreak in a teaching hospital. CMAJ 2003;168:1245-51.

9. King A. Hundreds of mental health workers offer free therapy to Ontario's frontline COVID-19 staff. CBC News 2020 Mar. 26. Available: www.cbc.ca/news/ canada/toronto/free-therapy-covid-19-health-workers-1.5510435 (accessed 2020 Apr. 6).

10. Vendeville G. As COVID-19 battle escalates, U of T students offer busy healthcare workers help on the home front. U of T News 2020 Mar. 19. Available: https://uoft.me/5f0 (accessed 2020 Apr. 6).

11. Edwardson L. University of Calgary medical students quadruple province's COVID-19 contact-tracing capacity. CBC News 2020 Mar. 25. Available: www. cbc.ca/news/canada/calgary/u-of-c-covid-19-contact-tracing-1.5509022?_vfz =medium\%3Dsharebar (accessed 2020 Apr. 6).

\section{Competing interests: None declared.}

This article has not been peer reviewed.

Affiliations: Division of General Internal Medicine (Wu, Gold), Division of Infectious Diseases (Gold) and Department of Psychiatry (Styra), University Health Network; Department of Medicine (Wu, Gold) and Department of Psychiatry (Styra), University of Toronto, Toronto, Ont.

Contributors: Peter Wu wrote the original draft of the manuscript. All of the authors contributed to the conception of the work, curated the data, critically revised the manuscript for important intellectual content, gave final approval of the version to be published and agreed to be accountable for all aspects of the work.

Correspondence to: PeterWu, peter.wu@uhn.ca 